\title{
REVISITING AN OLD FRIEND
}

by Bernard Dixon

$\mathrm{A}^{\mathrm{s}}$ s I was saying (Bio/Technology 4:1038, Dec. '86)...timeraveling 12 th century alchemists would find the biotransformations we do not make nowadays as interesting as those which are well established. I was discussing (remember?) the Finnish habit of exporting massive tonnages of barley while simultaneously importing massive tonnages of starch, sugar, ethanol, and soybean, and I went on to describe the country's new Rajamaki plant that will help to rationalize the picture.

Alchemists untutored in molecular biology might also puzzle over our continuing preoccupation with Escherichia coli as a workhorse for genetic engineering, at the expense of organisms with industrial value. Why, for example, given that certain species of Streptomyces produce almost two-thirds of our known natural antibiotics, have we not fashioned these organisms too by recombinant DNA techniques? One answer, of course, is that David Hopwood and his colleagues in Norwich, England have achieved just that. As spelled out in their "Genetic Manipulation of Streptomyces: A Laboratory Manual," Hopwood's team at the John Innes Institute has worked wonders in developing appropriate vectors, cloning genes, and evolving the now-practicable possibility of "hybrid antibiotics."

Nevertheless, by comparison with the elegant rapidity of $E$. coli manipulation, Streptomyces work is relatively laborious. Many recombinant plasmids are unstable, the organisms grow at the pace of a largo, and there has been only limited progress in developing methods such as cosmid cloning and transposon mutagenesis. In turn, Streptomyces engineers have managed to bridge the gap, literally, between their organism and its more accommodating cousin by fabricating shuttle plasmids that permit them to make constructions in $E$. coli and introduce these by transformation into organisms such as Streptomyces coelicolor and $S$. lividans. Again, however, this approach can be frustratingly time consuming. And it has little or no value as yet for researchers to work with many of the most handsome antibiotic producers.

May 1989 would be a good time for our alchemists to call, therefore, because they might well be able to witness the opening of a new phase in the industrial exploitation of Streptoymyces. It has been initiated as a result of some remarkable work by Philippe Mazodier and Charles Thompson at the Pasteur Institute in Paris, in collaboration with Ram Petter at Israel's Tel-Aviv University. Theirs is research which, at a stroke, spawns innumerable practical opportunities and prompts us to revise some of our ideas about horizontal gene flux in the soil.

The French/Israeli breakthrough originated in part with the recent demolition, by Patrick Trieu-Cuot and his colleagues at Pasteur, of the long-standing belief that plasmids do not transfer naturally between Gram-positive and Gram-negative bacteria (Trieu-Cuot, P., Carlier, C., Martin, P., and Courvalin, P., FEMS Microbiology Letters 48:289, 1987 and Trieu-Cuot, P., Carlier, C., and Courvalin, P., J. Bacteriology 170:4388, 1988). E. coli can indeed conjugate with organisms as different one from the other, and as distant in evolutionary terms, as Bacillus thuringiensis, Listeria monocytogenes, and Streptococcus lactis.

Perhaps the even more remote Streptomyces could be persuaded to conjugate with $E$. coli as well? Mazodier and his collaborators decided to experiment with pAT 187, the bifunctional vector which Trieu-Cuot et al. exploited in their studies. pAT 187 contains the origins of replication of pBR322 and the broad host-range streptococcal plasmid $\mathrm{pAM} \beta 1$. Its aminoglycoside phosphotransferase gene is expressed as a selectable marker in both Gram-positive and Gram-negative bacteria. But Mazodier's efforts to introduce it to $S$. lividans by conjugation from $E$, coli remained persistently unconsummated. The Pasteur Institute team decided, therefore, to construct two similar shuttle vectors in which pAM $\beta 1$ was replaced by the origin of replication of the Streptomyces plasmid p1J101.

As described in detail in a paper to appear shortly in the Journal of Bacteriology, the strategy worked. Both plasmids, which also carried the RK2(inc $P$ ) origin of transfer, were capable of conjugal transfer from $E$. coli to $S$. lividans, $S$. pristinaepolis, and $S$. viridochromogenes. The Pasteur Institute and Tel-Aviv University researchers believe this is the first recorded example of intergeneric conjugation between $E$. coli and filamentous bacteria of the actinomycete family.

Potential applications of the Paris/Tel-Aviv technique in achieving enhanced antibiotic production are obvious. But other possibilities present themselves. One is to instruct Streptomyces not only to generate novel proteins coded by foreign genes but also to secrete them conveniently into the culture medium. Another, explored in the ensuing book ("Biotechnology: Potentials and Limitations," Simon Silver (ed.), Springer-Verlag, p. 76), centers on the activation of "silent genes." Many actinomycetes, it seems, have the genetic capability to make more antibiotics than are detected in the laboratory-some being synthesized for only short periods of time as hormone-like triggers of sporulation and germination. Perhaps their production could be de-repressed by DNA fragments transferred from elsewhere?

Alongside such excitements, Mazodier's achievement also generates a more sobering thought. This study and the earlier work of Trieu-Cuot's group reinforce the ecological argument that we need to anticipate all realistically likely plasmid migrations following the release of engineered organisms into the environment. Once again, we see previously unrecognized possibilities for the transfer of genes into organisms distant-in space, time, and phylogeny-from the point of departure. Even allowing for arguments about selective advantage, this should make us cautious.

With this issue, Bernard Dixon rejoins Bio/Technology as a regular, monthly contributor. 